\title{
Discrimination of Retrograde from Anterograde Atrial Activation Using Intracardiac Electrogram Waveform Analysis
}

\author{
ROBERT D. THRONE, JANICE M. JENKINS, STUART A. WINSTON,* \\ CYNTHIA J. FINELLI, and LORENZO A. DICARLO, MD* \\ From the Medical Computing Laboratory, Department of Electrical Engineering and \\ Computer Science, University of Michigan, Ann Arbor, Michigan and \\ ${ }^{*}$ Cardiac Electrophysiology Laboratory, St. Joseph Mercy Hospital of the \\ Catherine McAuley Health Center, Ann Arbor, Michigan
}

THRONE, R.D., ET AL.: Discrimination of Retrograde from Anterograde Atrial Activation Using Intracardiac Electrogram Waveform Analysis. The prevention of pacemaker-mediated tachycardias requires a safe, reliable method for distinguishing retrograde from anterograde atrial activation by dual chamber pacemakers. In this study, a technique was developed to detect the morphological change that occurs in the waveform of the intra-atrial electrogram during retrograde atrial activation. The method employed for waveform analysis is based upon statistical correlation. In 19 patients undergoing electrophysiological studies, atrial electrograms were recorded from bipolar endocardial electrodes during sinus rhythm and 1:1 retrograde atrial depolarization while undergoing right ventricular pacing. Data were digitally sampled at 750,1,000, and 1,500 Hz. Templates of anterograde atrial depolarization were constructed by signal averaging waveforms from an initial sinus rhythm passage. These were used for analysis of anterograde depolarizations from a subsequent passage of sinus rhythm and a passage of known retrograde atrial depolarization. In all 19 cases, a patient-specific threshold could be derived to separate anterograde from retrograde atrial depolarizations using $1,000 \mathrm{~Hz}$ and $1,500 \mathrm{~Hz}$ sampling rates. However, at a sampling rate of $750 \mathrm{~Hz}$, separation of anterograde from retrograde atrial activation was possible in only 16/19 patients $(84 \%)$. We conclude that correlation waveform analysis of a suitably sampled atrial electrogram is a reliable method of discriminating retrograde atrial depolarization from anterograde atrial depolarization in intracardiac electrograms. (PACE, Vol. 12, October 1989)

pacemaker, pacemaker-mediated tachycardia, correlation waveform analysis, dual chamber pacemaker, tachycardia, tachyarrhythmia

\section{Introduction}

The introduction of dual chamber, dual sensing and inhibiting (DDD) pacemakers has allowed physicians more flexibility in the treatment of sinus slowing, sinus arrest, and atrioventricular

This work was partially supported by NSF grant No. ECS-8351215 and a grant from Medtronic, Inc.

Address for reprints: Lorenzo A. DiCarlo, M.D., Reichert Health Building R-3003, Catherine McAuley Health Center, P.O. Box 994, Ann Arbor, MI 48106.

Received March 30, 1989; revision June 14, 1989; accepted July 7, 1989 . block. A complication of this increased flexibility has been the occurrence of pacemaker-mediated tachycardia (PMT). PMT is most commonly initiated by atrial sensing of a retrogradely conducted ventricular depolarization followed by a sequentially initiated ventricular stimulus. ${ }^{1-5}$ PMT can also be initiated by other methods such as loss of atrial tracking, atrial premature depolarizations, or myopotential tracking. ${ }^{2,6}$

The most common method of preventing PMT is by programming the postventricular atrial refractory period (PVARP) to a value larger than the ventriculoatrial (VA) conduction time at the time of pacemaker implantation. ${ }^{2,3,6-11}$ Automatic 
prolongation of the PVARP after sensing a spontaneous premature or a paced ventricular premature depolarization (VPD) may inhibit atrial sensing of some retrograde atrial depolarizations; however, this method has the adverse effect of artificially restricting the upper limit of the atrial tracking rate. PMT may continue to occur if VA conduction time continues to increase when compared to initial measurements made at the time of implantation. ${ }^{1,6,12}$

Alternative methods have utilized detection of PMT in the presence of high ventricular rates and have been proposed for incorporation into DDD pacemakers. The stability of the retrograde ventriculoatrial (VA) interval during PMT has been examined, ${ }^{13}$ as has the effect of varying the AV delay and measuring the VA interval. ${ }^{3,6}$ Other proposed techniques of detecting PMT by DDD pacemakers have utilized extension of the PVARP after a predefined number of paced ventricular depolarizations above a predetermined rate $^{2,3}$ or automatically omitting a ventricular pacing stimulus. ${ }^{6}$ However, in some patients with true sinus or atrial tachycardia rather than PMT, the pacemaker may set up a long-short ventricular depolarization sequence causing torsade de pointes. ${ }^{14}$ In other cases PMT at rates below the threshold may not be detected. ${ }^{6}$

Some methods of detecting PMT have attempted to utilize the direct discrimination of retrograde atrial activation from anterograde atrial activation. Such methods have included the use of amplitude and slew rate, ${ }^{4,15-18}$ the gradient pattern detection (GPD) method, ${ }^{19-21}$ and combinations of time and frequency domain parameters. ${ }^{22}$ None of these methods have been found to be uniformly reliable.

Correlation waveform analysis has recently been found to be effective in discriminating the intracardiac ventricular electrograms which occur during ventricular tachycardia from those that occur during sinus rhythm. ${ }^{23}$ This method employs a statistical analysis of waveform morphology that produces a numerical value which specifies the amount of similarity or dissimilarity when two depolarizations are compared. The correlation coefficient is independent of the relative amplitudes of two signals, but is highly sensitive to subtle changes in shape. Any depolarization with a morphology identical or nearly iden- tical to a template derived during normal conduction will have a correlation coefficient of 1 , or close to 1 . As the difference between any depolarization and the template increases, the correlation coefficient decreases to a minimum value of -1 . While values near -1 reflect a high negative correlation in the classic sense, for the purposes of electrocardiographic waveform analysis such a value indicates a complete mismatch. This is because a waveform of opposite polarity from the normal depolarization, as detected by a closely spaced bipolar pair, indicates an entirely different direction of cardiac activation. Correlation waveform analysis has also been utilized for automated QRS analysis of surface ECG leads during cardiac monitoring. ${ }^{24,25}$ More recently, correlation waveform analysis has been applied to the esophageal electrogram and found effective in discrimination of retrograde from anterograde atrial depolarizations during continuous electrocardiographic monitoring. ${ }^{26,27}$

\section{Methods and Materials}

\section{Electrophysiology Study}

Bipolar atrial endocardial electrograms were recorded during elective clinical cardiac electrophysiology studies. Our patient population consisted of 12 men and 7 women (age 28 to 87 years). Patient data are given in Table I. None of the patients had dual atrioventricular nodal pathways or accessory atrioventricular connections. Patients were studied in a fasting postabsorptive state after sedation with 1-3 mg of intravenous medazolam. After administrating 1\% lidocaine for local anesthesia, two or three 7 French sidearm sheaths (Cordis Corp., Miami, FL, USA) were positioned in the right femoral vein using the Seldinger technique. Two or three 6 French quadrapolar electrode catheters with an interelectrode distance of $1 \mathrm{~cm}$ (USCI Division, C. R. Bard Inc., Billerica, MA, USA) were introduced and advanced under fluoroscopic guidance to the high right atrium or right atrial appendage, right ventricular apex, and, if three catheters were used, to the tricuspid valve for His bundle recording. All recordings were made with the patients lying supine. Immediately before programmed stimulation a 12-lead electrocardiogram was recorded during sinus rhythm. Atrial electrograms were 
Table I.

Patient Information

\begin{tabular}{|c|c|c|c|c|c|c|c|c|}
\hline Patient & Sex & Age & $\begin{array}{l}\text { Drug During } \\
\text { EPS }\end{array}$ & $\begin{array}{c}\text { Heart } \\
\text { Disease }\end{array}$ & $\begin{array}{c}\text { Atrial Electrode } \\
\text { Location }\end{array}$ & $1,500 \mathrm{~Hz}$ & $1,000 \mathrm{~Hz}$ & $750 \mathrm{~Hz}$ \\
\hline 1 & $F$ & 71 & Iso & None & RAA & + & + & + \\
\hline 2 & $M$ & 76 & None & None & RAA & + & + & + \\
\hline 3 & $M$ & 28 & None & None & RAA & + & + & - \\
\hline 4 & $\mathrm{~F}$ & 76 & None & None & RAA & + & + & + \\
\hline 5 & $M$ & 65 & None & CAD & RAA & + & + & + \\
\hline 6 & $M$ & 84 & None & None & RAA & + & + & + \\
\hline 7 & $M$ & 30 & None & None & RAA & + & + & + \\
\hline 8 & $\mathrm{~F}$ & 41 & None & None & RAA & + & + & + \\
\hline 9 & $\mathrm{M}$ & 65 & None & COPD & RAA & + & + & + \\
\hline 10 & $\mathrm{~F}$ & 43 & None & None & RAA & + & + & + \\
\hline 11 & $M$ & 44 & None & CAD & RAA & + & + & - \\
\hline 12 & $M$ & 74 & Ve & CAD & RAA & + & + & + \\
\hline 13 & $\mathrm{~F}$ & 72 & None & None & RAA & + & + & + \\
\hline 14 & $M$ & 87 & None & None & RAA & + & + & + \\
\hline 15 & $F$ & 67 & None & None & HRA & + & + & + \\
\hline 16 & $M$ & 56 & Qu Ve & None & HRA & + & + & - \\
\hline 17 & $M$ & 57 & None & None & HRA & + & + & + \\
\hline 18 & $\mathrm{~F}$ & 55 & Ep & None & HRA & + & + & + \\
\hline 19 & $M$ & 72 & En & None & HRA & + & + & + \\
\hline
\end{tabular}

En = Encainide; $\mathrm{Ep}=$ Epinephrine; Iso = Isopril; Qu = Quinidine; Ve = Verapamil $; \mathrm{CAD}=$ Cornary Artery Disease; $\mathrm{COPD}=$ Chronic Obstructive Pulmonary Disease; RAA = Right Atrial Appendage; HRA = High Right Atrium; + indicates positive separation, (- indicates no complete separation).

recorded on FM magnetic tape (Hewlett Packard 3968 and 3964A [Hewlett-Packard, San Diego, CA, USA]) from the bipolar endocardial electrodes positioned in either the right atrial appendage (14 patients) or the high right atrium (5 patients) using amplifiers (Siemens Mingograf-7 [Siemens-Elema, Solna, Sweden]) with filter settings of 0.5 to $500 \mathrm{~Hz}$. Tape speed was $3 \frac{3}{4}$ inches per second with a bandwidth of $0-1250 \mathrm{~Hz}$. Atrial electrograms were subsequently replayed and digitized on an IBM PC/AT compatible computer with a Tecmar Lab Master (Scientific Solutions, Inc., Solon, Ohio, USA) analog-to-digital system at sampling rates of $750 \mathrm{~Hz}, 1,000 \mathrm{~Hz}$, and $1,500 \mathrm{~Hz}$. Programs for digitization and subsequent waveform analysis were written in the $\mathrm{C}$ programming language and 8086 assembly language.

\section{Method of Analysis}

Data sets consisted of three 10 -second passages from each patient. Two separate passages were digitized from recordings made during sinus rhythm. A third passage was digitized from a segment recorded during pacing of the right ventricle at a rate sufficient to maintain 1:1 retrograde conduction to the atrium. The initial sinus rhythm (anterograde) passage was used to construct a template by signal averaging. The template was employed for subsequent comparison with a second separate sinus rhythm passage and a passage of retrograde atrial depolarization. A careful selection of window size ( 34 to $70 \mathrm{msec}$, mean $=44.5 \pm 8.3$ ) effectively excluded any local atrial repolarization in order to avoid the inclusion of injury current caused by temporary endocardial damage adjacent to the catheter. Depolarization was defined as beginning at the first deviation from baseline and ending at the instant repolarization began. These endpoints were determined visually prior to processing and were defined for each individual patient. A software trigger (digital differentiator) was used to detect automatically each of the sequential atrial waveforms. 


\section{Correlation Waveform Analysis}

The correlation coefficient is a statistical measure of the similarity of two waveforms. The value of the correlation coefficient $(\rho)$ falls between \pm 1 such that identical signals have a value of +1 , signals which are inverses of one another have a value of -1 , and dissimilar signals fall within that range. Mathematically, the correlation coefficient is defined as follows,

$$
\rho=\frac{\sum_{i=1}^{i=N}\left(T_{i}-\bar{T}\right)\left(S_{i}-\bar{S}\right)}{\sqrt{\sum_{i=1}^{i=N}\left(T_{i}-\bar{T}\right)^{2}} \sqrt{\sum_{i=1}^{i=N}\left(S_{i}-\bar{S}\right)^{2}}}
$$

where

$\mathrm{T}_{\mathrm{i}}=$ the template points.

$\mathrm{S}_{\mathrm{i}}=$ the signal points to be processed.

$\overline{\mathrm{T}}=$ the average value of the template points.

$\bar{S}=$ the average value of the signal points.

$\mathrm{N}=$ the number points in the template.

$\rho=$ the index of merit.

We may rewrite this as

$$
\begin{aligned}
\rho & =\sum_{i=1}^{i=N} \frac{\left(T_{i}-\bar{T}\right)}{\sqrt{\sum_{i=1}^{i=N}\left(T_{i}-\bar{T}\right)^{2}}} \frac{\left(S_{i}-\bar{S}\right)}{\sqrt{\sum_{i=1}^{i=N}\left(S_{i}-\bar{S}\right)^{2}}} \\
& =\sum_{i=1}^{i=N} t_{i} S_{i}
\end{aligned}
$$

with

$$
\begin{aligned}
t_{i} & =\frac{\left(T_{i}-\bar{T}\right)}{\sqrt{\sum_{i=1}^{i=N}\left(T_{i}-\bar{T}\right)^{2}}} \\
s_{i} & =\frac{\left(S_{i}-\bar{S}\right)}{\sqrt{\sum_{i=1}^{i=N}\left(S_{i}-\bar{S}\right)^{2}}}
\end{aligned}
$$

Notice that the correlation coefficient removes the mean value and scales both the template (T) and the signal (S), and thus is only measuring relative shape differences between signals.

\section{Triggering}

In computing the correlation coefficient between each depolarization and the template, a sliding window was used to effect the most precise alignment of each waveform. The correlation coefficient was computed by aligning the waveforms at the original trigger point and repeating the calculation in the following manner: the waveform under analysis was shifted in stepwise fashion to the left, recalculating the correlation coefficient at each sample point within a $50 \mathrm{msec}$ window, and similarly to the right for each sample point within a $50 \mathrm{msec}$ window. The maximum value of all such correlations within the sliding window was the indicator of best alignment and was taken to be the correlation coefficient for that depolarization. The potential advantage of this method is the creation of a best fit alignment for similar waveforms, yielding the highest possible correlation coefficient. The potential disadvantage of this method is the creation of artificially high correlation coefficients for dissimilar waveforms as well.

\section{Results}

Results of correlation waveform analysis for 19 patients are given in Table I for three separate sampling rates $(1,500 \mathrm{~Hz}, 1,000 \mathrm{~Hz}$, and $750 \mathrm{~Hz})$. Figures 1, 2 and 3 display the quantitative results for each of the three sampling rates. Results for each patient are displayed as a column located on the horizontal axis, while the ranges of the correlation coefficients are displayed along the vertical

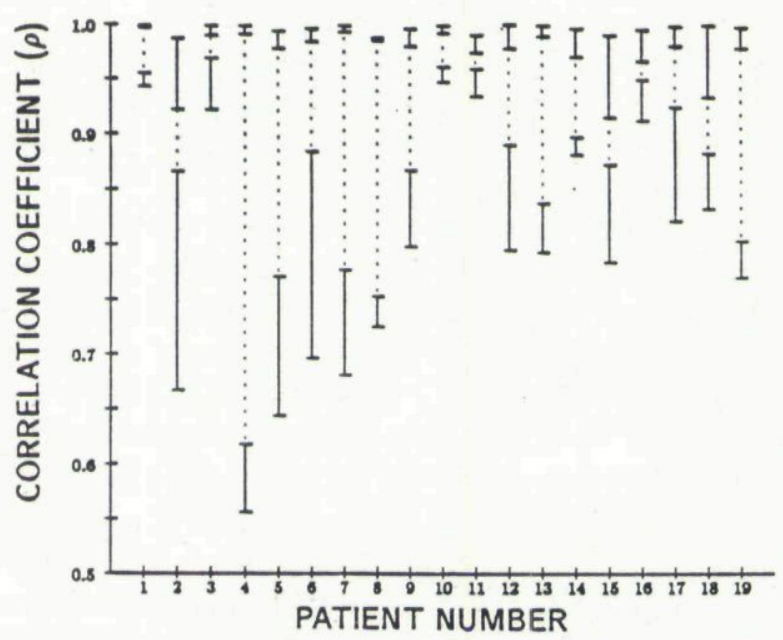

Figure 1. Plot of results of the correlation coefficient ( $\rho$ ) for discrimination of anterograde from retrograde atrial depolarizations for a sampling rate of $1,500 \mathrm{~Hz}$. Patients are listed by number on the horizontal axis. The range of correlation for each patient is seen in the vertical bars above the patient number. The upper entry shows the ranges of $\rho$ for anterograde (normal) atrial depolarizations; directly below are the ranges for retrograde atrial depolarizations. There is no overlap in ranges for any of the 19 patients. 


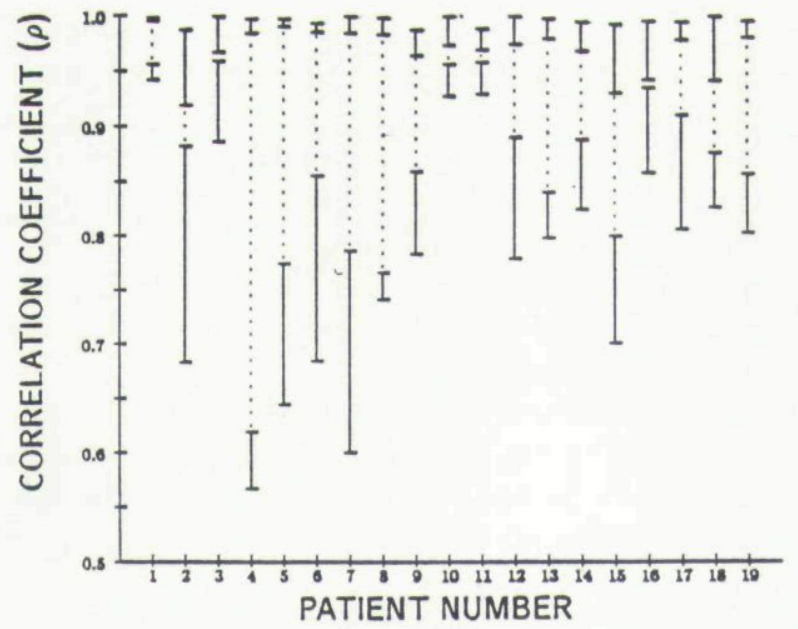

Figure 2. Plot of results of the correlation coefficient $(\rho)$ for discrimination of anterograde from retrograde atrial depolarizations for a sampling rate of $1,000 \mathrm{~Hz}$. Patients are listed by number on the horizontal axis. The range of correlation for each patient is seen in the vertical bars above the patient number. The upper entry shows the ranges of $\rho$ for anterograde (normal) atrial depolarizations; directly below are the ranges for retrograde atrial depolarizations. There is no overlap in ranges for any of the 19 patients.

axis. The range of the correlation coefficient for retrograde depolarization in each patient is displayed directly below the range for anterograde depolarization.

At sampling rates of $1500 \mathrm{~Hz}$ (Figure 1) and $1000 \mathrm{~Hz}$ (Figure 2) there is no overlap between ranges of anterograde and retrograde atrial depolarizations for an individual patient, indicating that a patient-specific threshold is possible for separation. However, the range of separation between classes is generally smaller at the $1000 \mathrm{~Hz}$ sampling rate than at the $1500 \mathrm{~Hz}$ sampling rate. Finally, at a $750 \mathrm{~Hz}$ sampling rate (Figure 3), there is overlap in the two classes of waveform for 3 of 19 patients. The separation range between anterograde and retrograde values for some patients is further decreased from those at either the $1500 \mathrm{~Hz}$ or $1000 \mathrm{~Hz}$ sampling rates.

Figures 4,5 , and 6 display the template, a typical anterograde atrial depolarization, and a typical retrograde atrial depolarization for patients 8,13 , and 5 , respectively. The paper speed is $200 \mathrm{~mm} / \mathrm{sec}$ and all three passages are displayed at the same gain setting for both retrograde and anterograde depolarizations. In Figure 4 (patient 8 ) the amplitude of the retrograde atrial depolarization is larger than the anterograde depolarization which would lead to a misdiagnosis by a system requiring that anterograde amplitude must be greater than retrograde. In Figure 5, (patient 13) the amplitude of the retrograde atrial depolarization is smaller than the anterograde depolarization. Finally, in Figure 6 (patient 5) both retrograde and anterograde atrial depolarizations are biphasic. However the correlation coefficient separates these signals at all three sampling rates.

\section{Discussion}

In 19 patients, correlation waveform analysis was shown to be capable of discriminating normal anterograde atrial depolarizations from retrograde atrial depolarizations induced by right ventricular pacing with $100 \%$ accuracy at a sampling rate of $1,000 \mathrm{~Hz}$ or higher. There was no global threshold which provided discrimination across

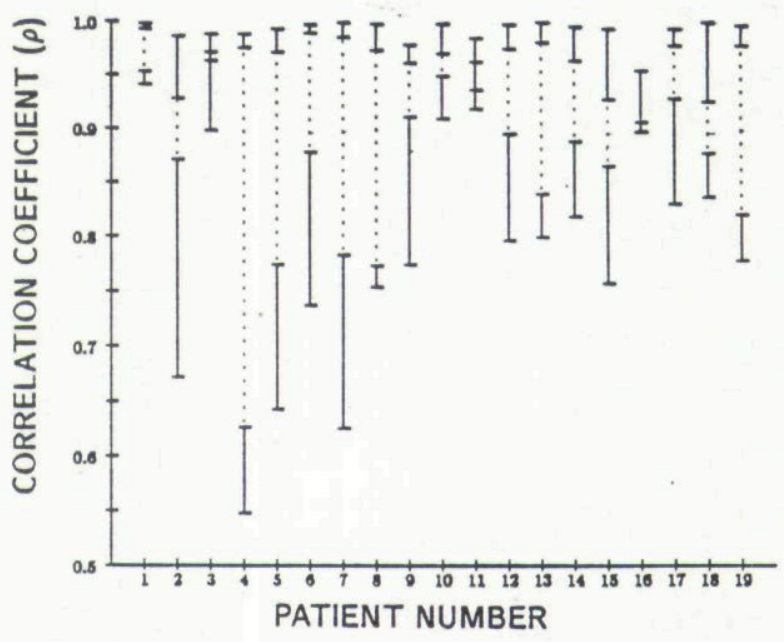

Figure 3. Plot of results of the correlation coefficient $(\rho)$ for discrimination of anterograde from retrograde atrial depolarizations for a sampling rate of $750 \mathrm{~Hz}$. Patients are listed by number on the horizontal axis. The range of correlation for each patient is seen in the vertical bars above the patient number. The upper entry shows the ranges of $\rho$ for anterograde (normal) atrial depolarizations, directly below are the ranges for retrograde atrial depolarizations. There is overlap in the ranges for 3 of the 19 patients $(16 \%)$. 


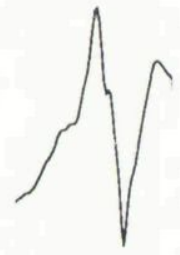

Template

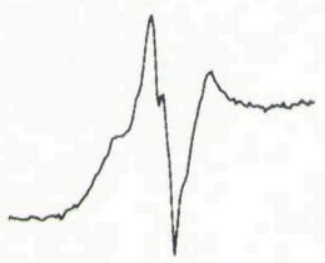

Anterograde

Depolarization

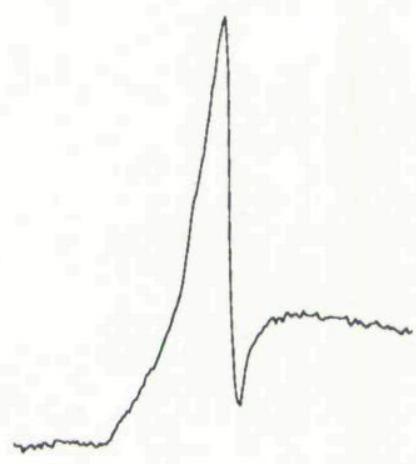

Retrograde
Depolarization

Figure 4. Examples of a template, a typical anterograde depolarization, and a typical retrograde depolarization (patient 8). Note that the retrograde depolarization has a significantly larger amplitude than the anterograde depolarization. However, the correlation coefficient separated anterograde from retrograde depolarizations for this patient at all three sampling rates $(750$, $1,000,1,500 \mathrm{~Hz})$.

all patients. Individual thresholds were required for each patient and were based on results of correlation waveform analysis of each patient's sinus rhythm passage.

The small window sizes used to capture only atrial depolarization and exclude injury current $(34-70$ msec, mean $=44.5 \pm 8.3)$, may have caused the results to become poorer as the sampling rate was decreased. The primary reason for the overlap in ranges of anterograde and retrograde atrial depolarizations at the $750 \mathrm{~Hz}$ sampling rate is probably not due to the high frequency content of the atrial depolarization. Rather, it appears that sampling rates must be high enough to include a suitable number of points in the template and waveforms under analysis to produce a reason-

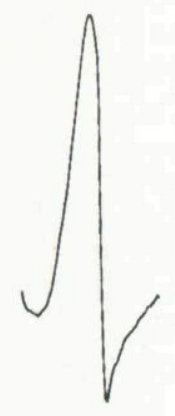

Template

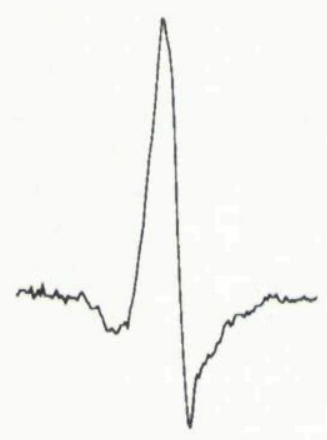

Anterograde

Depolarization

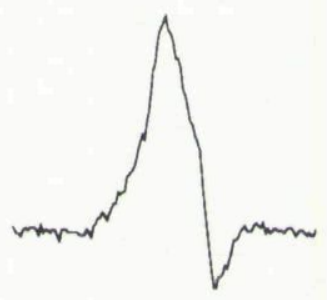

Retrograde

Depolarization

Figure 5. Examples of a template, a typical anterograde depolarization, and a typical retrograde depolarization (patient 13). Note that the retrograde depolarization has a significantly smaller amplitude than the anterograde depolarization. However, the correlation coefficient separated anterograde from retrograde depolarizations for this patient at all three sampling rates (750, $1,000,1,500 \mathrm{~Hz})$. 


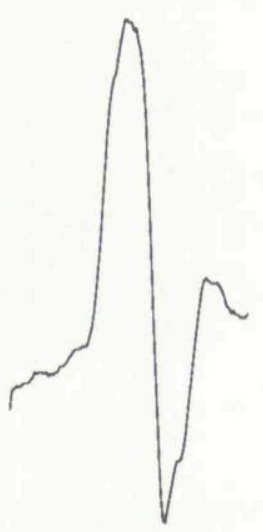

Template

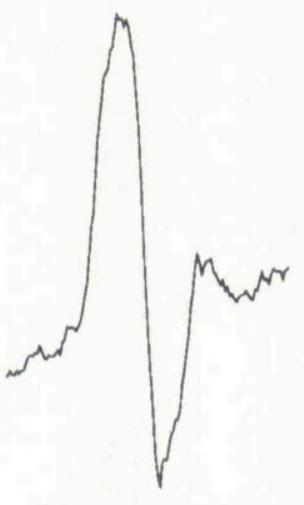

Anterograde

Depolarization

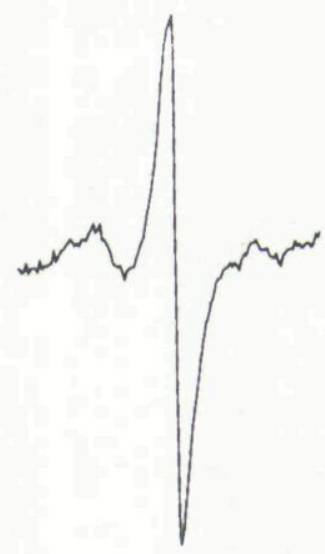

Retrograde
Depolarization

Figure 6. Examples of a template, a typical anterograde depolarization, and a typical retrograde depolarization (patient 5). Note that both the retrograde and the anterograde atrial depolarizations are biphasic and have similar amplitudes. The correlation coefficient can separate anterograde from retrograde depolarizations for this patient at all three sampling rates $(750,1,000,1,500$ $\mathrm{Hz}$ ).

able correlation analysis. This is especially true when there are subtle differences between anterograde and retrograde depolarization and the anterograde template size is small.

\section{Comparison with Previous Studies}

Direct discrimination of true retrograde from anterograde atrial depolarizations without regard to the timing of the waveform may be useful in prevention of PMT as well as in other arrhythmia diagnostic methods.

Timmis $^{22}$ examined various time and frequency domain criteria from the atrial electrograms of nine different patients using bipolar electrodes. These included peak-to-peak amplitude, duration, maximum slew rate, mean slew rate and polarity in the time domain, and maximum frequency, half-power frequency, Fourier amplitude peak, and peak frequency. No individual parameter was able to separate anterograde from retrograde depolarizations in more than four patients and at least three different parameters were needed for successful discrimination in $7 / 9$ patients. Wainwright ${ }^{19}$ and Davies ${ }^{20}$ recorded bipolar atrial electrograms from three atrial sites from ten patients and used sequential slew rate changes and successfully detected retrograde from anterograde depolarizations using the right atrial appendage electrogram in all ten patients. Retrograde conduction for $8 / 10(80 \%)$ could be detected from the high right atrial electrogram.

Pannizzo $^{15,16}$ measured amplitude, slew rate, and the configuration of retrograde and anterograde electrograms from the right atrial appendage in 34 patients ( 31 unipolar and 3 bipolar electrodes). While no single parameter could discriminate in all cases, the combined use of both amplitude and slew rate could differentiate anterograde from retrograde conduction in all 34 cases. However, this finding was not uniformly observed in subsequent studies. For example, Bernheim ${ }^{17}$ also used slew rate and amplitude measurements in 130 patients and found that reprogramming atrial sensitivity would have prevented PMT in only $60 \%$ of the patients. McAlister ${ }^{18}$ also analyzed both slew rate and amplitude. Using amplitude alone, retrograde activation could be distinguished in 68 out of 72 instances (23 patients), however, slew rates were not significantly different. In a subsequent study of 129 patients using unipolar atrial electrodes, McAlister ${ }^{4}$ found that in $81 \%$ of the patients the amplitude 
difference between the retrograde and anterograde atrial depolarization was at least $0.5 \mathrm{mV}$. These measurements were obtained at the time of implantation and could be expected to change as leads mature or during exercise. Thus, methods depending on amplitude alone may be of limited value. Ross ${ }^{28}$ studied the effects of exercise on the amplitude of the atrial electrogram from permanent leads in 11 patients and found an average amplitude decrease of $38.5 \%$ (range $18.9 \%$ to $77.8 \%$ ) during exercise. One important advantage of the correlation coefficient is that it measures only differences in waveshape and is, therefore, not dependent upon fluctuations in amplitude. ${ }^{29}$ (Fig. 4, 5, and 6.)

\section{Limitations}

This study was done using acute leads positioned during electrophysiological study and the recorded electrograms display characteristics not common to chronic leads. Motion artifact in acute leads may result in motion-related waveform changes. Results could be expected to improve with permanent leads since the wide range of anterograde depolarization correlation coefficients in patients $2,15,16$, and 18 may be due to motion of the acute leads.

For this study, the size of the template used for each patient was chosen individually to include only depolarization and not injury current or local atrial repolarization. Once injury current has subsided, fixed width templates which include repolarization in chronic leads may be feasible.

This study did not examine the effects of drugs on the morphology of the atrial depolarizations. To compensate for such changes, new an-

\section{References}

1. Fontain JM, Maloney JD, Castle LW, et al. Noninvasive assessment of ventriculo-atrial conduction and early experience with the tachycardia termination algorithm in pacemaker-mediated tachycardia. PACE 1986; 9:212-222.

2. Duncan JL, Clark MF. Prevention and termination of pacemaker-mediated tachycardia in a new DDD pacing system (Siemens-Pacesetter model 2010T). PACE 1988; 11:1679-1683.

3. Lamaison D, Girodo S, Limousin M, et al. A new algorithm for a high level of protection against terograde depolarization templates may occasionally need to be constructed. By deliberately pacing the ventricle to induce retrograde conduction and sampling one of the retrograde beats, the correlation coefficient between the current anterograde template and the retrograde depolarization can be computed, and if the discrimination is no longer sufficient, a new anterograde depolarization template could be constructed. It may also be possible to store a template from the retrograde as well as the anterograde depolarizations to determine directly if the depolarization in question is anterograde or retrograde.

\section{Conclusion}

Correlation waveform analysis appears to be a reliable method of discriminating anterograde from retrograde depolarization using bipolar endocardial atrial electrograms given a suitable sampling rate and may be feasible for incorporation in dual chamber pacemakers for detection of PMT. Correlation waveform analysis has previously been shown to separate ventricular electrograms of sinus rhythm depolarizations from those of ventricular tachycardia. ${ }^{23}$ Incorporation of a similar algorithm into dual chamber antitachycardia pacemakers may allow separation of both abnormal atrial and ventricular depolarizations and allow for improved tachycardia diagnosis.

Acknowledgments: The authors wish to express their appreciation to Kevin Price, B.S., Ryan Reeg, B.S., and Colleen Hoover, B.S., for their technical assistance in the cardiac electrophysiology laboratory, and Ms. Debbie Laird for preparation of this manuscript. 
maker mediated tachycardias: Techniques for induction, observation, and control. (abstract) J Am Coll Cardiol 1983; 1:674.

8. Svenson RH, Clark M, Hall D, et al. Analysis of manifest and latent retrograde conduction in patients with AV sequential pacemakers. Implications for pacer induced tachycardias. (abstract) J Am Coll Cardiol 1983; 1:674.

9. Fletcher RD, Cohen AI, Cutler DJ, et al. Dual chamber pacemakers as implanted electrophysiology laboratories. (abstract) Circulation 1984; 70:201.

10. Baker R, Sanders R, Calfee R, et al. Automatic measurement of retrograde conduction time by an implanted pacemaker. (abstract) PACE: Cardiostim 86 1986; 4:16.

11. den Dulk K, Wellens HJJ. Merits of anti-PCMT features. (abstract) PACE: Cardiostim 86 1986; 419.

12. Begemann MJS, Boute W. Automatic refractory period. PACE 1988; 11:1684-1686.

13. Kroiss D, Jacobson P, Limousin M, et al. Pacemaker-mediated tachycardia: New engineering solutions. (abstract) PACE: Cardiostim 86 1986; $4: 19$.

14. GN Kay, Plumb VJ, Arciniegas JG, et al. Torsade de pointes: The long-short initiating sequence and other clinical features: Observations in 32 patients. J Am Coll Cardiol 1983; 2:806-817.

15. Pannizzo F, Furman S. Automatic discrimination of retrograde $\mathrm{P}$ waves for dual chamber pacemakers. (abstract) J Am Coll Cardiol 1985; 5:393.

16. Pannizzo F, Amikam S, Bagwell P, et al. Discrimination of antegrade and retrograde atrial depolarization by electrogram analysis. Am Heart J 1986; 112:780-786.

17. Bernheim C, Markewitz A, Kemes BH. Can reprogramming of atrial sensitivity avoid an endless loop tachycardia? (abstract) PACE: Cardiostim 86 1986; 4:18.

18. McAlister H, Adelson R, Calderon E, et al. Atrial electrogram analysis: Antegrade vs retrograde. (abstract) PACE: Cardiostim 88 1988; 11:818.
19. Wainwright R, Davies W, Tooley M. Ideal atrial lead positioning to detect retrograde atrial depolarization by digitization and slope analysis of the atrial electrogram. PACE 1984; 7:1152-1158.

20. Davies DW, Wainwright RJ, Tooley MA, et al. Electrogram recognition by digital analysis: Relevance to pacemaker arrhythmia control? (abstract) J Am Coll Cardiol 1985; 5:507.

21. Davies DW, Wainwright RJ, Tooley MA, et al. Detection of pathological tachycardia by analysis of electrogram morphology. PACE 1986; 9:200-208.

22. Timmis GC, Westveer DC, Bakalyar DM, et al. Discrimination of antegrade from retrograde atrial electrograms for physiologic pacing. PACE 1988; 11:130-140.

23. Lin D, DiCarlo LA, Jenkins JM. Identification of ventricular tachycardia using intracavity ventricular electrograms: Analysis of time and frequency domain patterns. PACE 1988; 11:1592-1606.

24. Feldman CL, Amazeen PG, Klein MD, et al. Computer detection of ventricular ectopic beats. Comp Biomed Res 1971; 4:666-674.

25. Arzbaecher R, Biancalana P, Stibolt T, et al. Computer technique for detection of cardiac dysrhythmias. (abstract) J Assoc Advan Med Instrum 1971; 5:104.

26. Lin D, Jenkins JM, DiCarlo LA. Analysis of P-wave changes on the esophageal electrogram: A twolead arrhythmia analysis system using morphology and timing of both atrial and ventricular activation. IEEE Computers in Cardiology 1987; 83-86.

27. Lin D, Jenkins JM, DiCarlo LA. A practical model for automatic analysis of atrial and ventricular activation. (abstract) Circulation 1988; 78:556.

28. Ross BA, Zinner A, Ziegler V, et al. The effect of exercise on the atrial electrogram in humans. (abstract) J Am Coll Cardiol 1987; 9:32A.

29. Woodroofe M. Probability with Applications. McGraw-Hill, New York. 1975; 229. 
This document is a scanned copy of a printed document. No warranty is given about the accuracy of the copy. Users should refer to the original published version of the material. 